\title{
PLANET-PLANET ECLIPSE AND THE ROSSITER-MCLAUGHLIN EFFECT OF A MULTIPLE TRANSITING SYSTEM: JOINT ANALYSIS OF THE SUBARU SPECTROSCOPY AND THE KEPLER PHOTOMETRY
}

\author{
Teruyuki Hirano, ${ }^{1}$ Norio Narita, ${ }^{2}$ Bun'ei Sato, ${ }^{3}$ Yasuhiro H. Takahashi, ${ }^{2,4}$ Kento Masuda, ${ }^{1}$ Yoichi Takeda, ${ }^{2}$ \\ Wako Aoki, ${ }^{2}$ Motohide Tamura, ${ }^{2}$ And Yasushi Suto ${ }^{1,5}$
}

\begin{abstract}
We report a joint analysis of the Rossiter-McLaughlin (RM) effect with Subaru and the Kepler photometry for Kepler Object of Interest (KOI) 94 system. The system comprises four transiting planet candidates with orbital periods of 22.3 (KOI-94.01), 10.4 (KOI-94.02), 54.3 (KOI-94.03), and 3.7 (KOI-94.04) days from the Kepler photometry. We performed the radial velocity (RV) measurement of the system with the Subaru $8.2 \mathrm{~m}$ telescope on August 10, 2012 (UT), covering a complete transit of KOI-94.01 for $\sim 6.7$ hours. The resulting RV variation due to the RM effect spectroscopically confirms that KOI-94.01 is indeed the transiting planet, and implies that its orbital axis is well aligned with the stellar spin axis; the projected spin-orbit angle $\lambda$ is estimated as $-6_{-11}^{+13}$ deg. This is the first measurement of the RM effect for a multiple transiting system. Remarkably, the archived Kepler lightcurve around $\mathrm{BJD}=2455211.5$ (date in UT January 14/15, 2010) indicates a "double transit" event of KOI-94.01 and KOI-94.03, in which the two planets transit the stellar disk simultaneously. Moreover, the two planets partially overlap each other, and exhibit a "planet-planet eclipse" around the transit center. This provides a rare opportunity to put tight constraints on the configuration of the two transiting planets by joint analysis with our Subaru RM measurement. Indeed, we find that the projected mutual inclination of KOI-94.01 and KOI-94.03 is estimated to be $\delta=-1.15^{\circ} \pm 0.55^{\circ}$. Implications for the migration model of multiple planet systems are also discussed.

Subject headings: planets and satellites: individual (KOI-94) - planets and satellites: formation stars: rotation - techniques: radial velocities - techniques: spectroscopic
\end{abstract}

\section{INTRODUCTION}

The proximity of giant planets to their host stars implies that such planets have gone through so-called planetary migrations. The angle between the stellar spin axis and planetary orbital axis is an important probe to reveal the origin of planetary migrations (Queloz et al. 2000; Winn et al. 2005). While migrations at earlier stages due to interactions between planets and proto-planetary disk are supposed to result in smaller values for the spinorbit angles (Lubow \& Ida 2010), migrations caused by planet-planet scatterings or the Kozai cycle and subsequent tidal interactions with the host star could produce large spin-orbit angles (Marzari \& Weidenschilling 2002; Fabrvcky \& Tremaine 2007; Chatteriee et al.|2008; Nagasawa \& Ida 2011).

Observations of the Rossiter-McLaughlin (RM) effect have been the major channel to measure the skyprojected spin-orbit angles $\lambda$ (e.g., Ohta et al. 2005; Narita et al. 2007; Albrecht et al. 2012). With previous dedicated campaigns, last few years have witnessed an unexpectedly large fraction of spin-orbit misaligned systems, and even retrograde orbits with respect to the projected stellar equator have been reported (Narita et al.

\footnotetext{
Electronic address: hirano@utap.phys.s.u-tokyo.ac.jp

1 Department of Physics, The University of Tokyo, Tokyo, 1130033, Japan

2 National Astronomical Observatory of Japan, 2-21-1 Osawa, Mitaka, Tokyo, 181-8588, Japan

3 Department of Earth and Planetary Sciences, Tokyo Institute of Technology, 2-12-1 Ookayama, Meguro-ku, Tokyo 152-8551, Japan

4 Department of Astronomy, The University of Tokyo, Tokyo, 113-0033, Japan

${ }^{5}$ Department of Astrophysical Sciences, Princeton University, Princeton, NJ 08544
}

2009; Winn et al. 2009; Triaud et al. 2010), indicating that dynamical processes such as planet-planet scatterings are indeed responsible for some systems with close-in giant planets.

The spin-orbit angle for multiple transiting systems is particularly interesting. Such a multiplicity strongly suggests that the system has never experienced chaotic processes and rather results from a quiescent evolution history. A spin-orbit misalignment in a multiple planetary system implies that stellar obliquities can vary by processes which are unconnected to planetary migrations. (e.g., Lai et al. 2011; Rogers et al. 2012) Sanchis-Ojeda et al. (2012) measured the spin-orbit relation for the Kepler-30 system by a precise modeling of spot-crossing events during planetary transits and found a good spin-orbit alignment in the system, supporting the notion that the system has evolved quiescently in the disk.

In this letter, we focus on the KOI-94 system. This system, listed in the earliest Kepler Object of Interest (KOI) list (Borucki et al. 2011; Batalha et al. 2012), has four transiting planet candidates around a relatively bright late $\mathrm{F}$ star (with the Kepler magnitude of $K_{p}=12.205$ ). The orbital period and radius $R_{p}$ normalized by the stellar radius $R_{s}$ for each candidate are summarized in Table 1. Indeed KOI-94 is a remarkable system that experienced a "double transit" event due to the simultaneous transit of two planets on the stellar disk, and a "planetplanet eclipse" due to the partial overlap of the two planets during the transiting phase. As we describe below, a joint analysis of our Subaru radial velocity data and the Kepler lightcurve provides a unique opportunity to tightly constrain the configuration of the multi-transiting planetary system. 
TABLE 1

Properties of Planetary Candidates in KOI-94

\begin{tabular}{rcc}
\hline Candidate & Orbital Period (days) & $R_{p} / R_{s}$ \\
\hline \hline KOI-94.01 & $22.343000 \pm 0.000011$ & $0.06856 \pm 0.00012$ \\
94.02 & $10.423707 \pm 0.000026$ & $0.02544 \pm 0.00012$ \\
94.03 & $54.31993 \pm 0.00012$ & $0.04058 \pm 0.00013$ \\
94.04 & $3.743245 \pm 0.000031$ & $0.01045 \pm 0.00019$ \\
\hline
\end{tabular}

\section{OBSERVATIONS AND DATA REDUCTION}

Due to the long orbital distance of KOI-94.01 as a transiting planet, the transit duration is considerably long (about 6.7 hours), which makes it very challenging to observe a complete transit within a night by a ground-based telescope. A fortunate opportunity was given on 2012 August 10 (UT), when a complete transit of KOI-94.01 was observed by High Dispersion Spectrograph (HDS) installed on the Subaru $8.2 \mathrm{~m}$ telescope. We also obtained out-of-transit spectra on 2012 July 1, 2, 3, 4, August 8, 9 (UT), to determine the RV baseline and derive the RV semi-amplitude of the host star. In order to maximize the RV precision, we adopted the I2a setup, and utilized the image slicer (Tajitsu et al. 2012) with the Iodine cell for the precise RV calibration, achieving the spectral resolution of $R \sim 110,000$.

We reduced the raw data with the standard IRAF procedure and extracted 1-dimensional (1D) spectra. With a 20-minutes exposure, we typically achieved a signal-tonoise $(\mathrm{S} / \mathrm{N})$ of $80-90$ per pixel of the $1 \mathrm{D}$ spectra. A high $\mathrm{S} / \mathrm{N}$ template spectrum $(\mathrm{S} / \mathrm{N} \sim 150)$ of KOI-94 was obtained without the Iodine cell for the RV analysis. This template spectrum was then subject to deconvolution of the instrumental profile (IP), which was reproduced by taking the flat lamp spectrum transmitted through the Iodine cell. Using this deconvolved template representing the intrinsic stellar spectrum, we extracted the RV for each of the spectra by the RV analysis routine developed by Sato et al. (2002). The resulting relative RV's (after corrected for the motion of the Earth), along with their uncertainties are summarized in Table 2. The typical RV uncertainty is $\sim 10 \mathrm{~m} \mathrm{~s}^{-1}$.

\section{ANALYSIS AND RESULTS}

\subsection{Stellar Parameters}

Since KOI-94 is not a confirmed planetary system as of 2012 September and no spectroscopic measurement has been published, we ourselves estimate the basic stellar parameters from the template spectrum of KOI-94 used for the RV template. Following Takeda et al. (2002, 2005), we estimate the stellar atmospheric parameters $\left(T_{\text {eff }}\right.$, the surface gravity $\log g$, metalicity $[\mathrm{Fe} / \mathrm{H}]$, and microturbulence dispersion $\xi$ ), based on the equivalent widths of Fe I and Fe II lines located at $5000-7600 \AA$ wavelength region. The result of the measurement is shown in Table 3 (A).

We also extract the projected rotational velocity $V \sin I_{s}$ by modeling the line profiles of KOI-94's template. By convolving theoretical line profiles for the same type star with the rotation plus macroturbulence broadening kernel and the IP, we fit the line profiles around $6100 \AA$. In doing so, we assume that the macroturbulence dispersion $\zeta$ is $4.5 \pm 1.1 \mathrm{~km} \mathrm{~s}^{-1}$, based on the empirical
TABLE 2

Radial Velocities measured With Subaru/HDS. AN ARBITRARY RV OFFSET IS SUBTRACTED IN THE DATA.

\begin{tabular}{|c|c|c|}
\hline Time [BJD (TDB)] & Relative RV $\left[\mathrm{m} \mathrm{s}^{-1}\right]$ & Error $\left[\mathrm{m} \mathrm{s}^{-1}\right]$ \\
\hline 2456109.86607 & $\begin{array}{c}-16.9 \\
\end{array}$ & 14.1 \\
\hline 2456110.05250 & -14.0 & 16.1 \\
\hline 2456110.84846 & -13.4 & 13.1 \\
\hline 2456111.09425 & -24.2 & 10.7 \\
\hline 2456111.87151 & -35.5 & 11.1 \\
\hline 2456112.08319 & -8.2 & 10.9 \\
\hline 2456112.85317 & -32.7 & 12.7 \\
\hline 2456112.89785 & -31.4 & 12.1 \\
\hline 2456147.97040 & 0.8 & 9.8 \\
\hline 2456148.96781 & 9.7 & 13.4 \\
\hline 2456149.75308 & -5.2 & 9.6 \\
\hline 2456149.76802 & -13.6 & 9.5 \\
\hline 2456149.78275 & 4.1 & 8.9 \\
\hline 2456149.79747 & 14.2 & 8.7 \\
\hline 2456149.81220 & 0.5 & 9.6 \\
\hline 2456149.82692 & 15.1 & 10.1 \\
\hline 2456149.84163 & 12.5 & 9.6 \\
\hline 2456149.85635 & 16.2 & 9.6 \\
\hline 2456149.87107 & 13.5 & 9.7 \\
\hline 2456149.88579 & 9.4 & 9.6 \\
\hline 2456149.90050 & 9.9 & 8.8 \\
\hline 2456149.91522 & -7.1 & 9.8 \\
\hline 2456149.92994 & -13.6 & 9.4 \\
\hline 2456149.94466 & -16.4 & 10.0 \\
\hline 2456149.96175 & -20.8 & 9.3 \\
\hline 2456149.97647 & -34.6 & 8.6 \\
\hline 2456149.99119 & -45.8 & 9.4 \\
\hline 2456150.00591 & -44.6 & 9.2 \\
\hline 2456150.02063 & -43.2 & 9.2 \\
\hline 2456150.03536 & -44.0 & 9.2 \\
\hline 2456150.05008 & -28.3 & 9.8 \\
\hline 2456150.06480 & 9.9 & 10.4 \\
\hline 2456150.07952 & -3.6 & 10.1 \\
\hline
\end{tabular}

relation by Valenti \& Fischer (2005). The resulting bestfit value and its uncertainty for $V \sin I_{s}$ are also shown in Table 3(A). This procedure and validity of our measurement of $V \sin I_{s}$ are described by Hirano et al. (2012) in detail.

Given the atmospheric parameters, we estimate the mass and age of the host star. We here adopt the YonseiYale $\left(\mathrm{Y}^{2}\right)$ isochrone model (Yi et al. 2001). Based on this model, we compute the best-fit values and their uncertainties for the mass $M_{s}$, radius $R_{s}$, and age of KOI-94. Table 3 (A) also shows the result of those estimates.

\subsection{Modeling and Fitting of the Observed RV's}

The upper panel of Figure 1 plots the RV data phasefolded with the orbital period of KOI-94.01 (i.e., $\sim 22.34$ days), and the lower panel is the zoomed-in version around the transit phase after subtracting the orbit of KOI-94.01. The RV variation during the transit of KOI94.01 clearly indicates that the RM effect is securely detected, and that the planetary orbit is prograde with respect to the host star's equator.

Following Hirano et al. (2011a) and Narita et al. (2011), we fit the observed RV's assuming that the RV's can be approximated by the RV variation due to Keplerian motion of KOI-94.01 and the velocity anomaly due to the RM effect. Because of the lack of RV data points, we here neglect the impacts of KOI-94.02, 94.03, 94.04 on RV variations. Based on the transit depths of planetary candates reported by Kepler and using a 

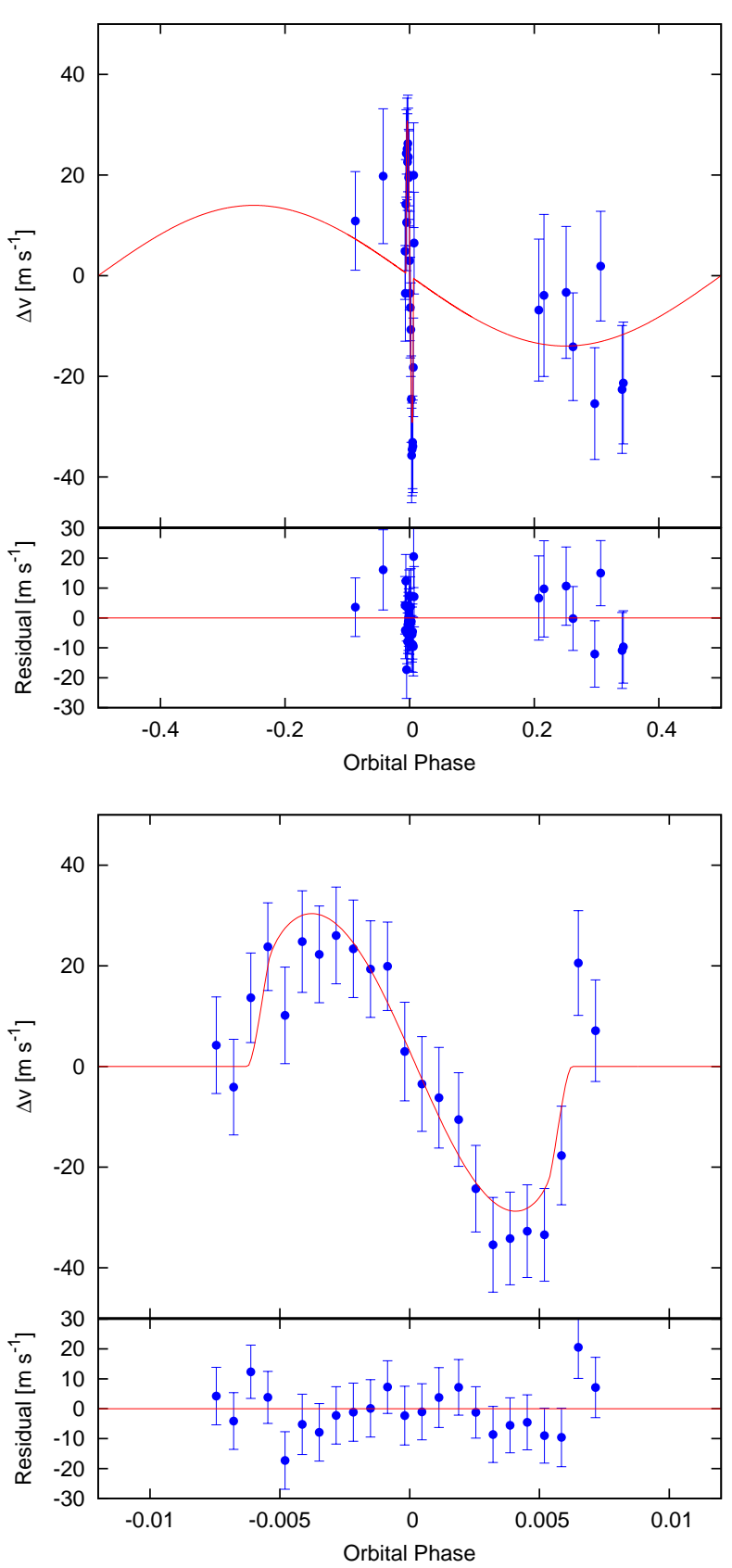

FIG. 1.- (Upper) Phase-folded RV data of KOI-94, obtained with Subaru/HDS. The best-fit model curve is shown in red. (Lower) The RV variation around the planetary transit of KOI-94.01 (blue) and its best-fit model (red solid line). The Keplerian orbit is subtracted from the RV's.

simple relation between the planet mass and radius, $M_{p} / M_{\oplus}=\left(R_{p} / R_{\oplus}\right)^{2.06}$, where $M_{\oplus}$ and $R_{\oplus}$ are respectively the Earth mass and radius (Lissauer et al. 2011), we roughly estimate the planet mass and compute the RV semi-amplitude exerted by each candidate; the ratios of RV semi-amplitudes for KOI-94.02, 03, and 04 to the semi-amplitude for KOI-94.01 are estimated to be 0.17 , 0.25 , and 0.04 , respectively. Considering that the maximum RV variation in Figure 1 is approximately $30 \mathrm{~m}$ $\mathrm{s}^{-1}$ and our error for each RV is no less than $10 \mathrm{~m} \mathrm{~s}^{-1}$, one-planet approximation for the RV variation of KOI94 is not a bad approximation. Precise measurements of
TABLE 3

System PARAMETERS

\begin{tabular}{lc}
\hline Parameter & Value \\
\hline \hline (A) Spectroscopic Parameters & \\
$T_{\text {eff }}(\mathrm{K})$ & $6116 \pm 30$ \\
$\log g(\mathrm{dex})$ & $-123 \pm 0.055$ \\
{$[\mathrm{Fe} / \mathrm{H}](\mathrm{dex})$} & $1.29 \pm 0.20$ \\
$\xi\left(\mathrm{km} \mathrm{s}^{-1}\right)$ & $7.33 \pm 0.32$ \\
$V \sin I_{s}\left(\mathrm{~km} \mathrm{~s}^{-1}\right)$ & $1.25_{-0.04}^{+0.03}$ \\
$M_{s}\left(M_{\odot}\right)$ & $1.61_{-0.12}^{+0.11}$ \\
$R_{s}\left(R_{\odot}\right)$ & $3.9_{-0.2}^{+0.3}$ \\
age $(\mathrm{Gyr})$ & $14.3 \pm 4.9$ \\
\hline$(\mathrm{B})$ Orbital and RM Parameters & $8.01_{-0.73}^{+0.72}$ \\
$K\left(\mathrm{~m} \mathrm{~s}^{-1}\right)$ & $-6_{-11}^{+13}$ \\
$V \sin I_{s}\left(\mathrm{~km} \mathrm{~s}^{-1}\right)$ & $-9.9_{-2.4}^{+2.5}$ \\
$\lambda\left(^{\circ}\right)$ & 0.80 \\
$\gamma_{\text {offset }}\left(\mathrm{m} \mathrm{s}{ }^{-1}\right)$ & \\
$\tilde{\chi}^{2}$ & \\
\hline$(\mathrm{C})$ Double Transit Parameters & $2455211.51363 \pm 0.00024$ \\
$T_{c}^{(1)}(\mathrm{BJD})$ & $0.10 \pm 0.06$ \\
$T_{c}^{(3)}(\mathrm{BJD})$ & $0.61 \pm 0.08$ \\
$u_{1}$ & $-1.15 \pm 0.55$ \\
$u_{2}$ & \\
$\delta\left(^{\circ}\right)$ &
\end{tabular}

the impacts KOI-94.02, 94.03, and 94.04 are beyond the scope of this letter and will be discussed together with further follow-up observations.

The RV variation including a transit phase is usually modeled as

$$
V_{\text {model }}=K[\cos (f+\varpi)+e \cos (\varpi)]+\Delta v_{\mathrm{RM}},
$$

where $K, e$, and $\varpi$ are the RV semi-amplitude, orbital eccentricity, and argument of periastron, respectively. Again, because of the lack of RV data points and the fact that KOI-94 is a densely packed multiple planetary system with two giant planets being within $\sim 0.3 \mathrm{AU}$ from the host star, we decided to fix the orbital eccentricity as zero, and put a rough constraint on the RV semiamplitude for KOI-94.01. Future long-term monitoring will the possibility of non-zero eccentricity. improve the analysis with non-vanishing eccentricity.

In order to model the RM velocity anomaly $\Delta v_{\mathrm{RM}}$, we employ the analytic formula by Hirano et al. (2011b). The analytic formula is derived for the case that RV's are extracted by fitting the stellar template spectrum taken outside of the transit to the distorted spectrum during a transit, and therefore is more suitable for our calibration of the RM velocity anomaly than the classical formula derived by taking the intensity-weighted center of the distorted line profile (e.g., Ohta et al. 2005). For the stellar parameters required for the RM formula, we adopt the macroturbulence dispersion of $\zeta=4.5 \mathrm{~km} \mathrm{~s}^{-1}$, and intrinsic Gaussian and Lorentzian line widths of $\beta=2.4$ $\mathrm{km} \mathrm{s}^{-1}$ and $\gamma=1.0 \mathrm{~km} \mathrm{~s}^{-1}$, respectively, based on the empirical estimates by Hirano et al. (2011b). As for the limb-darkening, we employ the quadratic limb-darkening law with $u_{1}=0.40$ and $u_{2}=0.31$ based on the table by Claret (2004). Also, we here adopt $R_{p} / R_{s}=0.06856$, $a / R_{s}=26.1$, and $I_{o}=89.33^{\circ}$ for the transit parameters of KOI-94.01, which are the values provided by the Kepler team.

We fit the observed RV's using Markov Chain Monte 
Carlo (MCMC) algorithm. The $\chi^{2}$ statistics in our case is

$$
\chi^{2}=\sum_{i} \frac{\left(V_{\text {model }, i}-V_{\mathrm{obs}, i}\right)^{2}}{\sigma_{i}^{2}}
$$

where $V_{\text {model, } i}$ and $V_{\text {obs }, i}$ are the $i$-th modeled and observed RV's, and $\sigma_{i}$ is its error. The remaining fitting parameters are $K$, the spin-orbit angle $\lambda, V \sin I_{s}$, and the RV offset (zero-point) $\gamma_{\text {offset }}$ of our dataset listed in Table 2. The resulting best-fit values and $1 \sigma$ errors are summarized in Table 3 (B). The red curves in Figure 1 indicate the best-fit models for the whole orbit (upper) and the RM effect (lower), respectively.

The best-fit value of $V \sin I_{s}$ purely estimated by the RM velocity amplitude $\left(V \sin I_{s}=8.01_{-0.73}^{+0.72} \mathrm{~km} \mathrm{~s}^{-1}\right)$ agrees well with the spectroscopically measured value $\left(V \sin I_{s}=7.33 \pm 0.32 \mathrm{~km} \mathrm{~s}^{-1}\right)$, validating our measurement and modeling of the RM effect. One concern in our analysis is that since we neglected the impacts of the other three planetary candidates, we might have obtained a systematically biased estimate for the RV semiamplitude $K$ of KOI-94.01. A spurious RV baseline during the transit phase leads to a systematically biased estimate for the spin-orbit angle $\lambda$, and sometimes makes an aligned system look misaligned, or vice versa. Thus, we perform the following test; we remove the RV data on the transit night, and fit the remaining RV's with $K$ and $\gamma_{\text {offset }}$ being free. Then, using the best-fit value of $K$, we fit the RV's during the transit night in order to derive $\lambda$ and $V \sin I_{s}$ for the fixed $K$. This treatment can remove possible systematics caused by instrumental effects, the impact of other planets, and/or non-zero eccentricity. As a result, we find $V \sin I_{s}=8.02_{-0.74}^{+0.86} \mathrm{~km}$ $\mathrm{s}^{-1}$ and $\lambda=-10_{-11}^{+13} \mathrm{deg}$, implying a good spin-orbit alignment again. Based on this test, we conclude that the measurement of the spin-orbit angle $\lambda$ is robust and the orbital axis of KOI-94.01 is almost parallel to the projected stellar spin axis.

Substituting the best-fit value of $K$ and our estimate for the stellar mass $M_{s}$, we obtain $\sim 0.23 M_{J}$ for the planet mass of KOI-94.01. This mass is slightly small compared to the theoretical expectation for a planet having a radius of $\sim 9.25 R_{\oplus}$ (Mordasini et al. 2012), but is rather similar to low-density planets like Kepler-9c (Holman et al. 2010). In our analysis, however, we completely neglected the impacts of the other three planet candidates so that this estimate of the planet mass more or less has systematics. Again, a long-term RV monitoring is required in order to put tight constraints on masses and eccentricities of all the planet candidates in this system.

\section{DISCUSSION AND SUMMARY}

While we have seen that KOI-94.01's orbital axis is aligned with the stellar rotation axis, it is not evident that the orbits of the other planet candidates are also neatly aligned with the stellar spin. Fortunately, however, we found a lucky lightcurve in which a "double transit" event is observed in Kepler's public lightcurve. The upper panel of Figure 2 shows the normalized lightcurve of KOI-94 around BJD $=2455211.5$. In this figure, the first transit event starts around BJD $=2455211.35$, and after a while, it shows the second, deeper dimming. According to the public Kepler planet candidate ephemeris,

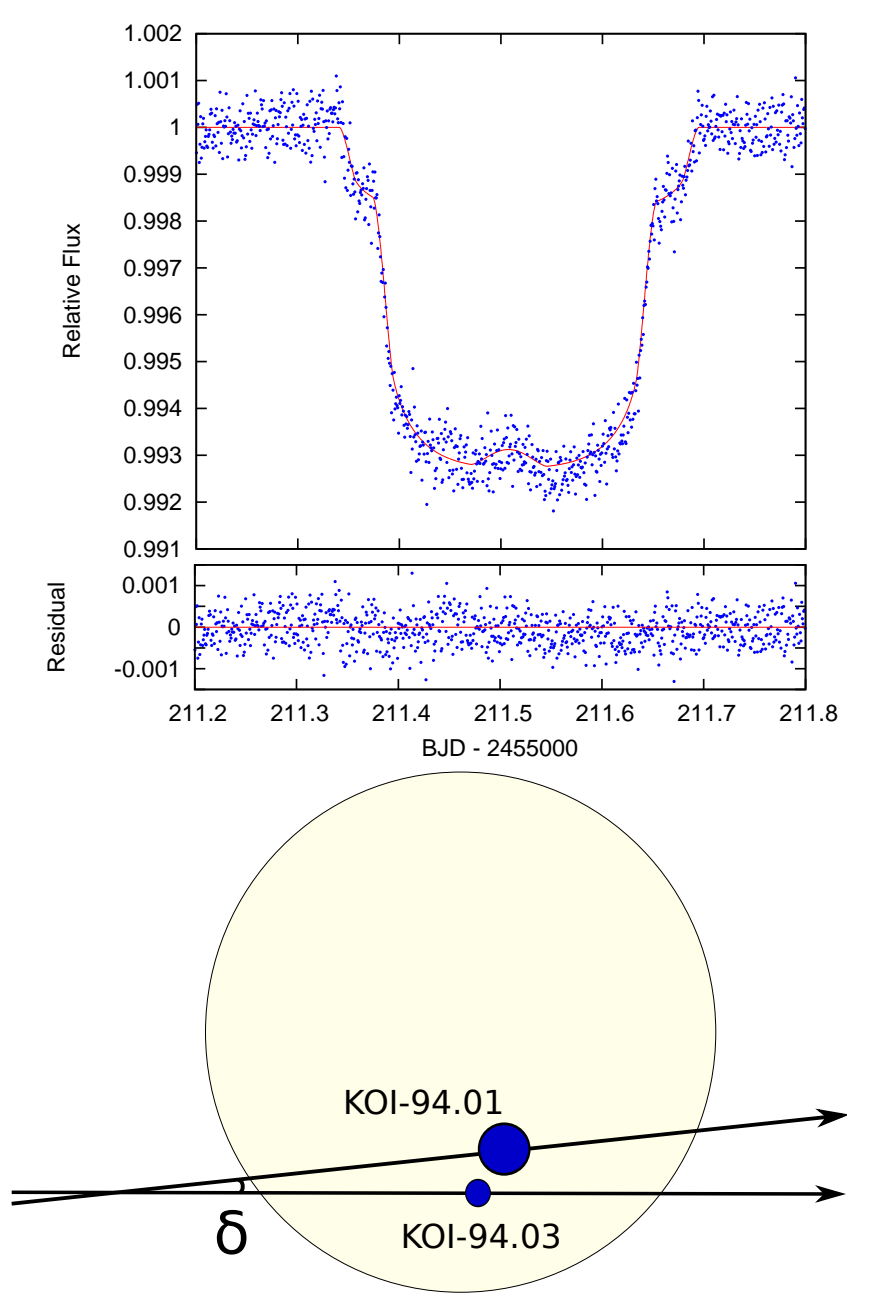

FIG. 2.- (Upper) The public lightcurve taken by Kepler around $\mathrm{BJD}=2455211.5$. In addition to the simultaneous double transit event, there is a bump around the transit center of KOI-94.01, which most likely represents the planet-planet eclipse. (Lower) The definition of the mutual inclination $\delta$ between KOI-94.01 and 94.03. The orbits of the two planets are projected onto the sky plane. This is just a schematic description and does not reflect the the real planet sizes and angle between the planetary orbits.

the first shallow dimming represents the transit of KOI94.03 and the second deeper one corresponds to a transit of KOI-94.01. Interestingly enough, there is a bump around the transit center with height of $\sim 0.0003$ in terms of the relative flux. Similar events have been frequently seen when the transiting planet crosses a starspot (Sanchis-Ojeda et al. 2011). We checked the out-oftransit lightcurve (long-cadence data) and computed the periodogram in order to look for a signal induced by starspots. Consequently, we found a weak peak around the period of $\sim 11$ days. However, the flux variability in the lightcurve (as is defined in Hirano et al. 2012) is about $0.02 \%$ so that the size of the bump seen in Figure 2 cannot be explained by a spot-crossing event. Considering the timing and size of the bump, the most likely scenario would be the eclipse of the planet by another transiting planet (planet-planet eclipse, also called "mutual event", Ragozzine \& Holman 2010). Since KOI94.03 has the longer orbital distance from the host star, a part of the inner planet, KOI-94.01, is occulted by the outer transiting planet, KOI-94.03, during the eclipse 
event represented by the bump.

Since the impact parameters of the two transiting planets are fixed from the transit photometries, the only parameter to determine the relative planetary orbits on the stellar disk and describe the bump in Figure 2 is the mutual inclination between the two transiting planets. If the mutual inclination of the two planets is considerably large, we expect no planet-planet eclipse. The timing and shape of the anomalous bump in Figure 2 put a strong constraint on the mutual inclination.

We define the mutual inclination projected onto the sky as $\delta$ (see the lower panel of Figure 2) and model the anomalous bump by a simple geometric calculation as a function of time and $\delta$. The mutual inclination $\delta$ sensitively changes the duration of the planet-planet eclipse, and can be constrained by the shape of the bump around the bottom of the transit lightcurve; the resulting bump is longer for nearly prograde $(\delta \approx 0)$, and shorter for retrograde planets $(\delta \approx \pi)$. By modeling the double transit event as a sum of two single transit lightcurves and the bump function (which is expressed analytically as an area fraction of the overlapping region by the two transiting planets), we fit the observed lightcurve by MCMC algorithm. The free parameters in our fit are the mid-transit times $T_{c}^{(1)}$ and $T_{c}^{(3)}$ for KOI-94.01 and KOI-94.03, the limb-darkening parameters $u_{1}$ and $u_{2}$ for the quadratic limb-darkening law ${ }^{6}$, and the projected mutual inclination $\delta$. The other transit parameters (i.e., $R_{p} / R_{s}, a / R_{s}$, and $i_{o}$ ) for both of 94.01 and 94.03 are fixed at the values delivered by the Kepler team assuming circular orbits for both of the candidates. Table 3 (C) shows the best-fit values and their uncertainties of our fitting. The red solid line in the upper panel of Figure 2 indicates the best-fit model and the residual of observed data from the best-fit model is shown at the bottom. The orbital planes of the two transiting planets are remarkably well aligned, which means that host star's spin axis and planetary axes of both KOI-94.01 and 94.03 are all well aligned.

Like the Kepler-30 system, the spin-orbit alignment in the KOI-94 system implies that those planets in this multiple transiting system have experienced a quiescent migration process rather than having been carried to the present locations by dynamical scattering processes or long term perturbations by outer objects. This has also ruled out the possibility that an earlier magnetic interaction between the star and disk causes a gradual misalignment of the stellar spin from the disk plane (Lai et al. 2011) at least for this system. On the other hand, our result should reinforce the hypothesis that spin-orbit misalignments are only seen for "isolated" hot Jupiters, which may have migrated by chaotic processes (Sanchis-Ojeda et al. 2012).

Finally, we note that KOI-94 has the effective temperature of $T_{\text {eff }}=6116 \pm 30 \mathrm{~K}$, which suggests that the star most likely has a convective envelope rather than being fully covered by a thick radiative envelope as seen for massive (hotter) stars (Pinsonneault et al. 2001). Thus, this system does not provide us the chance to test the recent theoretical work by Rogers et al. (2012), stating that the stellar spins of massive stars with radiative envelopes and convective cores may spontaneously change directions due to the internal gravity waves (IGW) generated around the border between the radiative envelope and convective core, regardless the presence of planets. A future observation of the RM effect for a multiple transiting system with a massive host star will confirm or refute this intriguing scenario.

This letter is based on data collected at Subaru Telescope, which is operated by the National Astronomical Observatory of Japan. We are very grateful to the support for our Subaru HDS observations by Akito Tajitsu, a support scientist for the Subaru HDS. We express special thanks to Hiroki Harakawa and Eric Gaidos for exchanging the observation time for obtaining additional RV data, and to Roberto Sanchis-Ojeda and Atsushi Taruya for a helpful discussion and important suggestions on this manuscript. The data analysis was in part carried out on common use data analysis computer system at the Astronomy Data Center, ADC, of the National Astronomical Observatory of Japan. T.H. is supported by Japan Society for Promotion of Science (JSPS) Fellowship for Research (DC1: 22-5935). T.H. and N.N. acknowledge a support by NINS Program for Cross-Disciplinary Study. N.N. is supported by Grant-in-Aid for Research Activity Start-up No. 23840046. M.T. is supported by the Ministry of Education, Science, Sports and Culture, Grantin-Aid for Specially Promoted Research, 22000005. Y.S. gratefully acknowledges support from the Global Collaborative Research Fund "A World-wide Investigation of Other Worlds" grant and the Global Scholars Program of Princeton University, the Grant-in-Aid No. 24340035 by JSPS. We acknowledge the very significant cultural role and reverence that the summit of Mauna Kea has always had within the indigenous people in Hawai'i. Finally, we deeply acknowledge the referee, Joshua Winn, for his important suggestions to improve the manuscript.

\section{REFERENCES}

Albrecht, S., et al. 2012, ApJ, 757, 18

Batalha, N. M., et al. 2012, ArXiv e-prints

Borucki, W. J., et al. 2011, ApJ, 728, 117

Chatterjee, S., Ford, E. B., Matsumura, S., \& Rasio, F. A. 2008, ApJ, 686, 580

Claret, A. 2004, A\&A, 428, 1001

Fabrycky, D., \& Tremaine, S. 2007, ApJ, 669, 1298

Hirano, T., Narita, N., Sato, B., Winn, J. N., Aoki, W., Tamura, M., Taruya, A., \& Suto, Y. 2011a, PASJ, 63, L57

Hirano, T., Sanchis-Ojeda, R., Takeda, Y., Narita, N., Winn, J. N., Taruya, A., \& Suto, Y. 2012, ApJ, 756, 66

Hirano, T., Suto, Y., Winn, J. N., Taruya, A., Narita, N., Albrecht, S., \& Sato, B. 2011b, ApJ, 742, 69

Holman, M. J., et al. 2010, Science, 330, 51

Lai, D., Foucart, F., \& Lin, D. N. C. 2011, MNRAS, 412, 2790
Lissauer, J. J., et al. 2011, ApJS, 197, 8

Lubow, S. H., \& Ida, S. 2010, Planet Migration, ed. Seager, S., 347-371

Marzari, F., \& Weidenschilling, S. J. 2002, Icarus, 156, 570

Mordasini, C., Alibert, Y., Benz, W., Klahr, H., \& Henning, T. 2012, A\&A, 541, A97

Nagasawa, M., \& Ida, S. 2011, ApJ, 742, 72

Narita, N., Hirano, T., Sato, B., Harakawa, H., Fukui, A., Aoki, W., \& Tamura, M. 2011, PASJ, 63, L67

Narita, N., Sato, B., Hirano, T., \& Tamura, M. 2009, PASJ, 61, L35

Narita, N., et al. 2007, PASJ, 59, 763

Ohta, Y., Taruya, A., \& Suto, Y. 2005, ApJ, 622, 1118

Pinsonneault, M. H., DePoy, D. L., \& Coffee, M. 2001, ApJ, 556, L59 
Queloz, D., Eggenberger, A., Mayor, M., Perrier, C., Beuzit, J. L., Naef, D., Sivan, J. P., \& Udry, S. 2000, A\&A, 359, L13

Ragozzine, D., \& Holman, M. J. 2010, ArXiv e-prints

Rogers, T. M., Lin, D. N. C., \& Lau, H. H. B. 2012, ArXiv e-prints

Sanchis-Ojeda, R., Winn, J. N., Holman, M. J., Carter, J. A., Osip, D. J., \& Fuentes, C. I. 2011, ApJ, 733, 127

Sanchis-Ojeda, R., et al. 2012, Nature, 487, 449

Sato, B., Kambe, E., Takeda, Y., Izumiura, H., \& Ando, H. 2002, PASJ, 54, 873

Tajitsu, A., Aoki, W., \& Yamamuro, T. 2012, PASJ, 64, 77

Takeda, Y., Ohkubo, M., \& Sadakane, K. 2002, PASJ, 54, 451
Takeda, Y., Ohkubo, M., Sato, B., Kambe, E., \& Sadakane, K. 2005, PASJ, 57, 27

Triaud, A. H. M. J., et al. 2010, A\&A, 524, A25+

Valenti, J. A., \& Fischer, D. A. 2005, ApJS, 159, 141

Winn, J. N., Johnson, J. A., Albrecht, S., Howard, A. W., Marcy, G. W., Crossfield, I. J., \& Holman, M. J. 2009, ApJ, 703, L99 Winn, J. N., et al. 2005, ApJ, 631, 1215

Yi, S., Demarque, P., Kim, Y.-C., Lee, Y.-W., Ree, C. H., Lejeune, T., \& Barnes, S. 2001, ApJS, 136, 417 